\title{
Silica nanoparticles increase human adipose tissue-derived stem cell proliferation through ERKI/2 activation
}

This article was published in the following Dove Press journal:

International Journal of Nanomedicine

24 March 2015

Number of times this article has been viewed

\author{
$\mathrm{Ki}$ Joo Kim ${ }^{1,2}$ \\ Young Ae Joe ${ }^{3}$ \\ Min Kyoung Kim ${ }^{1,2}$ \\ Su Jin Lee' \\ Yeon Hee Ryu ${ }^{1,2}$ \\ Dong-Woo Cho ${ }^{4,5}$ \\ Jong Won Rhie ${ }^{1,2}$
}

'Department of Plastic Surgery, College of Medicine, ${ }^{2}$ Department of Molecular Biomedicine, ${ }^{3}$ Cancer Research Institute and Department of Medical Lifescience, The Catholic University of Korea, Seoul, Republic of Korea; ${ }^{4}$ Department of Mechanical Engineering, Pohang University of Science and Technology, Gyeongbuk, Republic of Korea; ${ }^{5}$ Department of Integrative Bioscience and Bioengineering, Pohang University of Science and Technology, Gyeongbuk, Republic of Korea
Correspondence: Jong Won Rhie Department of Plastic Surgery, College of Medicine, The Catholic University of Korea, 222 Banpo-dearo, Seocho-gu, Seoul, 137-70I, Republic of Korea Tel +82222587525

Fax +8225947230

Email rhie@catholic.ac.kr
Background: Silicon dioxide composites have been found to enhance the mechanical properties of scaffolds and to support growth of human adipose tissue-derived stem cells (hADSCs) both in vitro and in vivo. Silica (silicon dioxide alone) exists as differently sized particles when suspended in culture medium, but it is not clear whether particle size influences the beneficial effect of silicon dioxide on hADSCs. In this study, we examined the effect of different sized particles on growth and mitogen-activated protein kinase signaling in hADSCs.

Methods: Silica gel was prepared by a chemical reaction using hydrochloric acid and sodium silicate, washed, sterilized, and suspended in serum-free culture medium for 48 hours, and then sequentially filtered through a $0.22 \mu \mathrm{m}$ filter (filtrate containing nanoparticles smaller than $220 \mathrm{~nm}$; silica NPs). hADSCs were incubated with silica NPs or $3 \mu \mathrm{m}$ silica microparticles (MPs), examined by transmission electron microscopy, and assayed for cell proliferation, apoptosis, and mitogen-activated protein kinase signaling.

Results: Eighty-nine percent of the silica NPs were around 50-120 nm in size. When hADSCs were treated with the study particles, silica NPs were observed in endocytosed vacuoles in the cytosol of hADSCs, but silica MPs showed no cell entry. Silica NPs increased the proliferation of hADSCs, but silica MPs had no significant effect in this regard. Instead, silica MPs induced slight apoptosis. Silica NPs increased phosphorylation of extracellular signal-related kinase (ERK)1/2, while silica MPs increased phosphorylation of p38. Silica NPs had no effect on phosphorylation of Janus kinase or p38. Pretreatment with PD98059, a MEK inhibitor, prevented the ERK1/2 phosphorylation and proliferation induced by silica NPs.

Conclusion: Scaffolds containing silicon dioxide for tissue engineering may enhance cell growth through ERK1/2 activation only when NPs around 50-120 nm in size are included, and single component silica-derived NPs could be useful for bioscaffolds in stem cell therapy.

Keywords: particle, ceramic, biomaterial, mesenchymal stem cells, ERK

\section{Introduction}

A number of bioceramic mixtures have been evaluated in terms of their ability to promote cellular attachment and induction of osteogenic differentiation. There have been many reports of enhanced cellular bioactivity using biosilicates $\left(\mathrm{P}_{2} \mathrm{O}_{5}-\mathrm{Na} \mathrm{Na}_{2} \mathrm{O}-\mathrm{CaO}-\right.$ $\left.\mathrm{SiO}_{2}\right),{ }^{1}$ calcium-magnesium-silicate ceramics, ${ }^{2}$ calcium-silica ceramics, ${ }^{3}$ and bioactive glass (Ca-Sr-Na-Zn-Si). ${ }^{4}$ Mesoporous silica particles are also being used for controlled release scaffolds, ${ }^{5}$ and many bioceramics include a silica component.

Silica is a ceramic material that has been successfully used for regeneration of bone and cartilage. It exists naturally in small quantities in a water-soluble form, but is most often found in an insoluble form. ${ }^{6}$ Orthosilicic acid $\left(\mathrm{Si}[\mathrm{OH}]_{4}\right)$, a soluble form of silicon, does not affect the synthesis of type I collagen for bone tissue regeneration, whereas an 
insoluble form, silica $\left(\mathrm{SiO}_{2}\right)$, enhances the synthesis of type I collagen and alkaline phosphate. ${ }^{7}$ Silica-based ceramics are mechanically strong and suitable for bone regeneration. ${ }^{8}$ Silicon nitride is resistant to bacterial infection during bone regeneration, ${ }^{9}$ and silicon increases the proliferation and osteogenic differentiation of osteoblast-like cells. ${ }^{10}$ In addition, silica-based bioglass ceramics and biosilicates enhance bone-like structure formation. ${ }^{1,2}$ A mixture of silicon and calcium, ${ }^{3,11}$ or mixtures with various other materials, including zinc and magnesium, ${ }^{12}$ improve both gene expression and biological performance.

Adult mesenchymal stem cells can be isolated from various tissues, including bone marrow, umbilical cord blood, cord matrix, and adipose tissues. Recently, adipose tissue-derived stem cells (ADSCs) have been used in the tissue engineering field owing to their abundance and ease of separation. ${ }^{13,14}$ ADSCs have been used for tissue regeneration to induce osteogenic differentiation in cell therapy. ADSCs combined with an hydroxyapatite bioceramic scaffold can construct vascularized tissue-engineered bone. ${ }^{15}$ ADSCs strongly induce osteogenic differentiation when applied to multiple-combination, such as electromagnetic field, ultrasound, and cyclic strain. ${ }^{16}$

The extracellular signal-related kinase (ERK)1/2 signaling pathway is a mitogen-activated protein kinase (MAPK) pathway that transduces signals from receptors and endocytosis at the cell surface. ${ }^{17,18}$ ERK1/2 signaling is involved in biopolymer-induced proliferation and differentiation of stem cells. The ERK1/2 and Wnt signaling pathways are also involved in biomaterial-induced osteogenic differentiation. ${ }^{19}$ Signaling by Janus kinase (JNK), a member of the MAPK family, activates cell death from hypoxia/reoxygenation condition in cultured ADSCs. ${ }^{20,21}$ Apoptosis of ADSCs driven by miR10b and miR23b expression occurs via the p38 signaling pathway. ${ }^{22}$

Most studies have evaluated bioceramic materials containing multiple components with silica. No evidence regarding silica alone as a biomaterial component is available. Given that mixtures of ceramic compounds are used to construct bioscaffolds, interference in the components of the mixture may occur, and their effects on the cellular response could differ. It is necessary to evaluate cellular responses to single-component scaffolds. When chemically synthesized silica is suspended in culture medium, it contains differently sized particles. It is unclear whether particle size affects the effect of silica on human ADSCs (hADSCs). Therefore, in this study, we examined whether single-component silica containing different sized silica particles, ie, nanoparticles
(NPs) or microparticles (MPs), has a differential effect on proliferation and MAPK signaling in hADSCs.

\section{Materials and methods Cell preparation and culture}

hADSCs were isolated and cultured as described previously. ${ }^{23}$ Informed consent was obtained from patients in accordance with the requirements of the institutional review board at Seoul St Mary's Hospital, Republic of Korea (KC11TNMS0095). Raw lipoaspirates from patients were washed thoroughly with sterile phosphate-buffered saline (Life Technologies, Carlsbad, CA, USA), minced, and then digested with $0.1 \%$ type I collagenase (Sigma-Aldrich, St Louis, MO, USA) in phosphate-buffered saline for 30 minutes at $37^{\circ} \mathrm{C}$ in a $5 \% \mathrm{CO}_{2}$ atmosphere with gentle agitation. The collagenase was inactivated with an equal volume of culture medium containing 10\% fetal bovine serum (Wisent Inc, St Bruno, QC, Canada). Cells were harvested by centrifugation at $250 \times g$ for 5 minutes and filtered through $100 \mu \mathrm{m}$ mesh to remove debris. The cells were plated onto conventional culture plates in Dulbecco's modified Eagle's medium (DMEM, Life Technologies) containing 10\% fetal bovine serum and $1 \%$ antibiotic/antimycotic (Life Technologies) at $37^{\circ} \mathrm{C}$ in a humidified atmosphere containing $5 \% \mathrm{CO}_{2}$. The culture medium was changed every 3 days.

\section{Preparation of medium containing different sized particles}

Silica gel was prepared via a chemical neutralization reaction by mixing hydrochloric acid with a solution of reagent grade sodium silicate $\left(\mathrm{SiO}_{2} 26.5 \%, \mathrm{Na}_{2} \mathrm{O} 10.6 \%\right.$, and $\mathrm{H}_{2} \mathrm{O} 62.9 \%$, Sigma Aldrich; Figure 1A and B). Silica gel of $6 \mathrm{~g}$ synthesized from the sodium silicate solution of $23 \mathrm{~g}$ according to the law of conservation of mass. After thorough washing with phosphate-buffered saline and sterilization, the gel was added to $100 \mathrm{~mL}$ of DMEM for 48 hours. The gel-containing medium was filtered through a $0.22 \mu \mathrm{m}$ filter (Thermo, Waltham, MA, USA). Filtered DMEM have got silica NPs (silica NP medium). Silica MPs purchased from Ditto Technology (Anyang City, Korea) were suspended same concentration of silica NP medium in DMEM (silica MP medium). The particle concentration in each type of medium was measured using an inductively coupled plasma optical emission spectrometer (Varian 710-ES, Varian, Melbourne, Australia). The size of the silica NPs was determined by transmission electron microscopy (JEM1010, JEOL Ltd, Tokyo, Japan). Basic fibroblast growth factor (Sigma-Aldrich) was used as a positive control. 
A

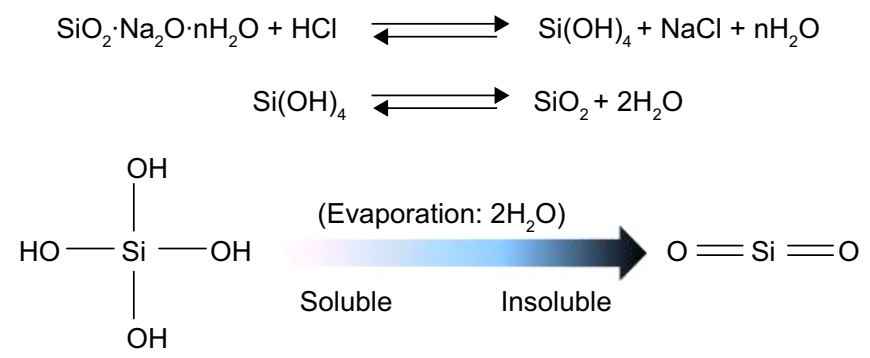

Orthosilicic acid

Silicon dioxide

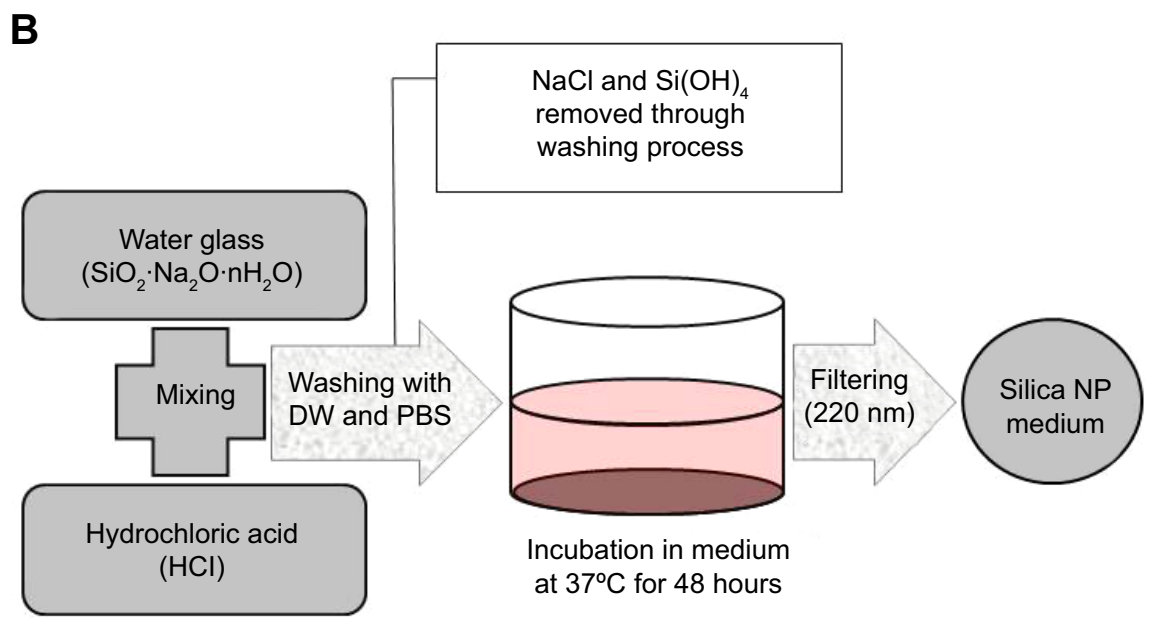

Figure I Preparation of silica NPs.

Notes: (A) Conversion of orthosilicic acid to silicon dioxide. (B) Manufacturing process for silica by neutralization reaction. The silica NP medium was prepared by incubating silica in culture medium for 48 hours and then by filtering through a $0.2 \mu \mathrm{m}$ filter.

Abbreviations: DW, distilled water; PBS, phosphate-buffered saline; NPs, nanoparticles.

\section{Total DNA assay}

To evaluate cell proliferation and apoptosis, total DNA content was measured using the CyQUANT ${ }^{\circledR}$ cell proliferation assay kit (Life Technologies), according to the manufacturer's protocol. Cells were cultured in a 96-well plate at $37^{\circ} \mathrm{C}$ in a $5 \% \mathrm{CO}_{2}$ atmosphere for 1,3 , and 5 days at a density of 3,000 cells/well with DMEM (with $1 \%$ fetal bovine serum) containing silica NPs and MPs, and then washed with phosphate-buffered saline to remove nonadherent cells. Cells were mixed in lysis buffer for an hour and CyQUANT GR dye was mixed with lysis buffer in a 96-well plate, which was then incubated for 10 minutes. Fluorescent signals were detected with excitation at $480 \mathrm{~nm}$ and emission at $520 \mathrm{~nm}$ using a spectrofluorometer (Gemini, SpectroMAX, Sunnyvale, CA, USA).

\section{Annexin $\mathrm{V}$ analysis by flow cytometry}

Cells were cultured in a 12-well plate at a density of 30,000 cells/ well for confluency. They were then treated with $1 \times$ phosphatebuffered saline and transferred to a fluorescence-activated cell sorting tube with phosphate-buffered saline at a concentration of 25,000 cells $/ \mathrm{mL}$ and centrifuged at $1,000 \times g$. The cells were stained with Annexin V and propidium iodide (BioBud Inc, Seoul, Korea) according to the manufacturer's protocol. The cells were mixed with $500 \mu \mathrm{L}$ of $1 \times$ binding buffer and incubated with $1.25 \mu \mathrm{L}$ of Annexin $\mathrm{V}$ in the dark for 15 minutes. After treatment, the cells were collected, mixed with $500 \mu \mathrm{L}$ of $1 \times$ binding buffer and $10 \mu \mathrm{L}$ of propidium iodide, and analyzed immediately by flow cytometry (BD FACSCalibur ${ }^{\mathrm{TM}}$, BD Biosciences, San Jose, CA, USA).

\section{Assay of DNA fragmentation by DAPI staining}

Cells were cultured in a six-well plate at $37^{\circ} \mathrm{C}$ in a $5 \% \mathrm{CO}_{2}$ atmosphere for 3 days at a density of 70,000 cells/well. The medium was then replaced with DMEM containing silica NPs or MPs. Cultured cells with NPs or MPs were incubated for 7 days at $37^{\circ} \mathrm{C}$ in a $5 \% \mathrm{CO}_{2}$ atmosphere. Culture medium was removed and DAPI reagent (Vector Laboratories Inc, Burlingame, CA, USA) was added. The stained cells were 
observed using a fluorescence microscope (Axiovert200, Carl Zeiss, Oberkochen, Germany).

\section{Protein extraction and Western blotting}

Cultured cells were washed twice with ice-cold phosphatebuffered saline, and $100 \mu \mathrm{L}$ of T-PER protein extraction reagent (Thermo Scientific, Rockford, IL, USA) was added to the culture dish. After scraping, the contents of the dish were transferred to a $1.5 \mathrm{~mL}$ tube and shaken at $4^{\circ} \mathrm{C}$ for a minute. The samples were centrifuged for 15 minutes at $4^{\circ} \mathrm{C}$ and $11,000 \times g$. The supernatant was transferred to a $1.5 \mathrm{~mL}$ flash tube. Quantitative analysis was performed using the Bradford assay (Bio-Rad, Hercules, CA, USA). For Western blotting, equal amounts of protein $(15 \mu \mathrm{g})$ were electrophoresed in $10 \%$ sodium dodecyl sulfate-acrylamide gels. The proteins were transferred from the gel to a $0.45 \mu \mathrm{m}$ nitrocellulose transfer membrane (Whatman GmbH, Dassel, Germany) using an electrical transfer system. Nonspecific binding was blocked with 5\% skim milk (BD Biosciences) in TBST buffer (5 mM Tris-HCl [pH 7.6], $136 \mathrm{mM} \mathrm{NaCl}$, $0.1 \%$ Tween 20 ) for an hour. The blots were then incubated with the appropriate primary antibody (dilution 1:1,000) for 24 hours at $4^{\circ} \mathrm{C}$, after which they were washed three times with $1 \times$ TBST. Next, the blots were incubated for 2 hours at room temperature with a secondary antibody $(1: 5,000)$. The blots were washed three times with $1 \times$ TBST and developed using electrochemiluminescence Western detection reagents (GE Healthcare, Little Chalfont, UK). Blots were analyzed using a luminescent image analysis instrument (Fuji Photofilm, Tokyo, Japan). We used the Multi Gauge program (version 3.0) for quantitative assays. The primary antibodies used were specific for ERK1/2 (Cell Signaling Technology Inc, Danvers, MA, USA), $p$-ERK1/2 (Cell Signaling), JNK (Cell Signaling), $p$-JNK (Cell Signaling), p38 (Cell Signaling), p-p38 (Cell Signaling), and actin (Sigma-Aldrich). The secondary antibodies were anti-rabbit and anti-mouse immunoglobulin G-horseradish peroxidase conjugates (both from Santa Cruz Biotechnology Inc, Santa Cruz, CA, USA).

\section{Treatment with PD98059}

Cells were grown to $70 \%$ confluency, after which the medium was replaced with fetal bovine serum-free DMEM. After 24 hours, the cells were treated with PD98059 $(40 \mu \mathrm{M}$; Cell Signaling Technology Inc) for 30 minutes, followed by incubation for 10 minutes with DMEM containing silica NPs or MPs. The negative control group was not treated with silica-containing medium, and the positive control group was treated with basic fibroblast growth factor.

\section{Results \\ Evaluation of silica NPs and MPs}

Silica (99.8\% purity) was prepared as reported previously (Figure S1A) and was synthesized in a dose-dependent manner form the ratio of sodium silicate reagent (Figure S1B). The silica was then incubated in serum-free DMEM at $37^{\circ} \mathrm{C}$ for 48 hours and filtered through $0.22 \mu \mathrm{m}$ mesh to obtain the silica NP fraction (Figure 1A and B). When we examined each type of medium by transmission electron microscopy, $89 \%$ of particles in the silica NP medium were around $50-120 \mathrm{~nm}$ in size (Figure 2A). Figure S2A and B show the respective size distributions for the silica NPs and MPs. Silica MPs obtained commercially was suspended in DMEM (Figure 2B). Seventy-eight percent of the silica MPs was about 2-4 $\mu \mathrm{m}$ in size. When hADSCs were incubated with each type of silica particle-containing medium for 24 hours, treatment of silica MPs showed no visible particles inside the cells like untreated cells (Figure 2C and D), whereas silica NPs entered the cytosol of cells (Figure 2E and F; Figure S3A and B). Some silica NPs were observed in endocytosed vacuoles in the cytosol of hADSCs. We therefore concluded that only silica NPs entered the cytosol, and in part through endocytosis.

\section{Effect of silica NPs and MPs on proliferation and apoptosis}

Since silica composites are known to promote proliferation of mesenchymal stem cells, we tested whether differentially sized particles containing a single component, ie, silica, affect proliferation of mesenchymal stem cells. After incubation with the particles for one, 3 , and 5 days, hADSCs underwent quantification of their DNA content using the CyQUANT cell proliferation assay kit (Figure 3A). The same amount of silicon was used in each culture to compare the cellular activity of the particles. Silica NPs increased cell proliferation significantly, but silica MPs showed no stimulation of cell proliferation, even at day 5 .

Next, we tested whether the particles have differential effects on apoptosis of hADSCs. Analysis of apoptotic cells by Annexin V staining confirmed that hADSCs exposed to silica MPs undergo apoptosis $(6.49 \%)$ in a $1 \%$ serum medium, albeit at a low degree. In contrast, silica NPs had no effect on apoptosis $(0.16 \%$; Figure $3 \mathrm{~B})$. DNA fragmentation, detected by DAPI staining, was observed only in silica MPtreated cells and not in silica NP-treated cells (Figure 3C). Thus, we concluded that silica NPs stimulated proliferation of ADSCs, whereas silica MPs induced apoptosis, albeit at a very low level. 

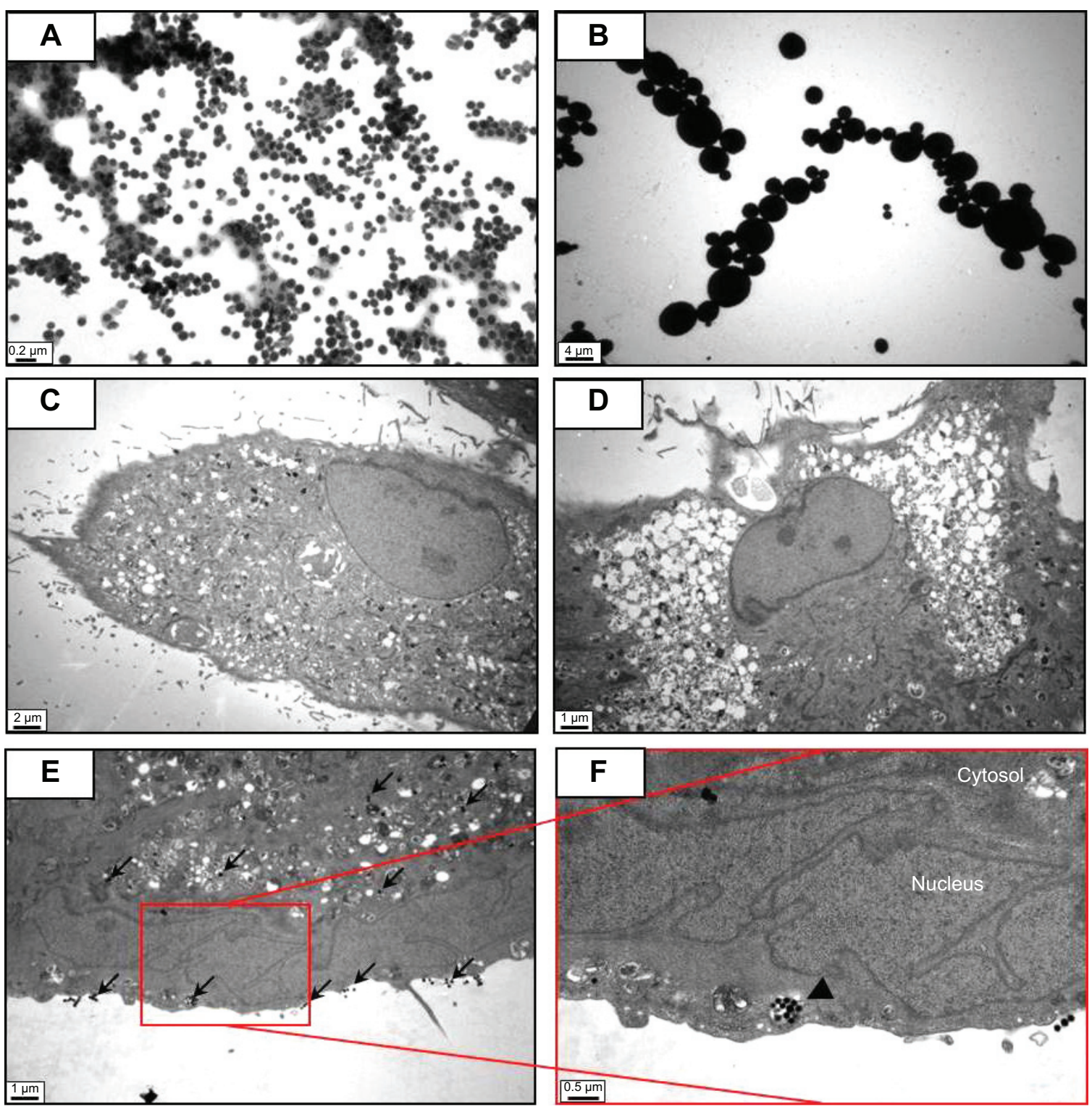

Figure 2 Transmission electron microscopy of different sized particles and observation of treated cells.

Notes: (A) NP and (B) MP media were observed by transmission electron microscopy. Adipose tissue-derived stem cells were incubated in each type of medium for 24 hours and then observed by transmission electron microscopy. Representative images are shown for (C) untreated cells, (D) silica MP-treated cells, and (E, F) silica NPtreated cells. Black arrows indicate particles and the triangle indicates a vesicle.

Abbreviations: NP, nanoparticle; MP, microparticle.

\section{Effects of silica NPs and MPs on MAPK signaling}

We examined whether differently sized silica particles affected MAPK signaling. After ADSCs were exposed to silica NP or MP medium for 10 to 60 minutes, we measured the phosphorylation levels of ERK1/2, p38, and JNK (Figure 4A). As shown in Figure 4B, the silica NP medium markedly increased the phosphorylation of ERK1/2 after 10 minutes, and phosphorylation decreased gradually thereafter. The silica NP medium did not significantly affect $\mathrm{p} 38$ phosphorylation, but the silica MP medium showed increased levels of the phosphorylated form of p38 (Figure 4C). However, there was no change in phosphorylation of $\mathrm{JNK}$ in either the silica NP or MP medium (Figure S4B).

To confirm the involvement of the ERK signaling pathway, cells were pretreated with PD98059 before silica NP medium was added. PD98059 indeed abolished phosphorylation of ERK $1 / 2$ in both basic fibroblast growth factor-treated and silica NP-treated cells. PD98058 also blocked the hADSC proliferation induced by silica NPs (Figure 4D). We concluded that silica NPs activated ERK1/2, whereas silica MPs activated p38. 
A

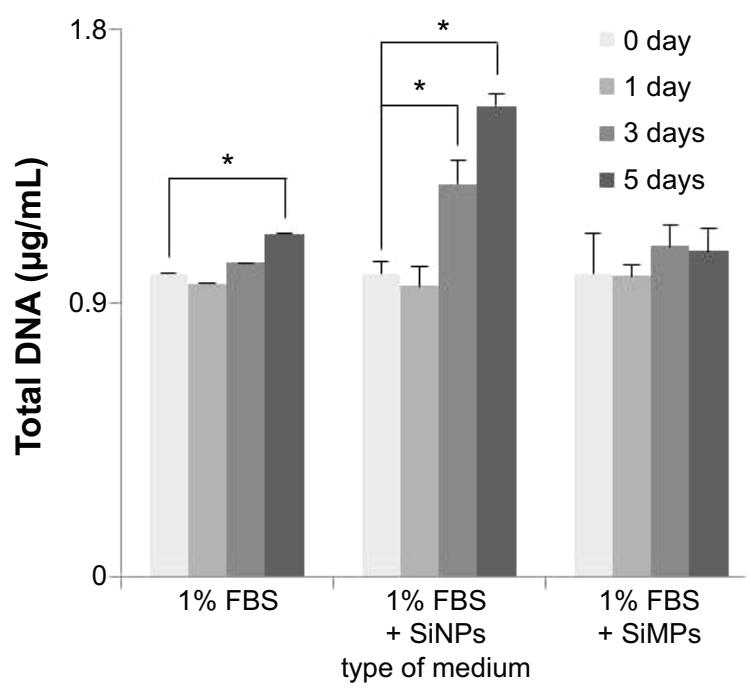

C

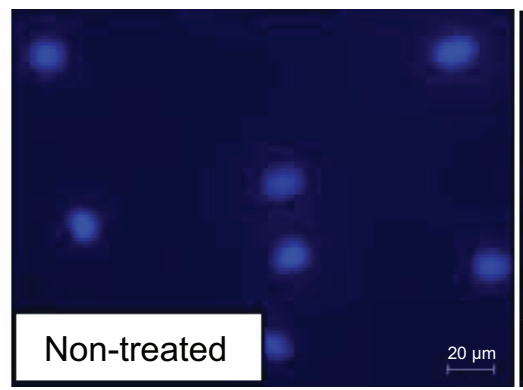

B

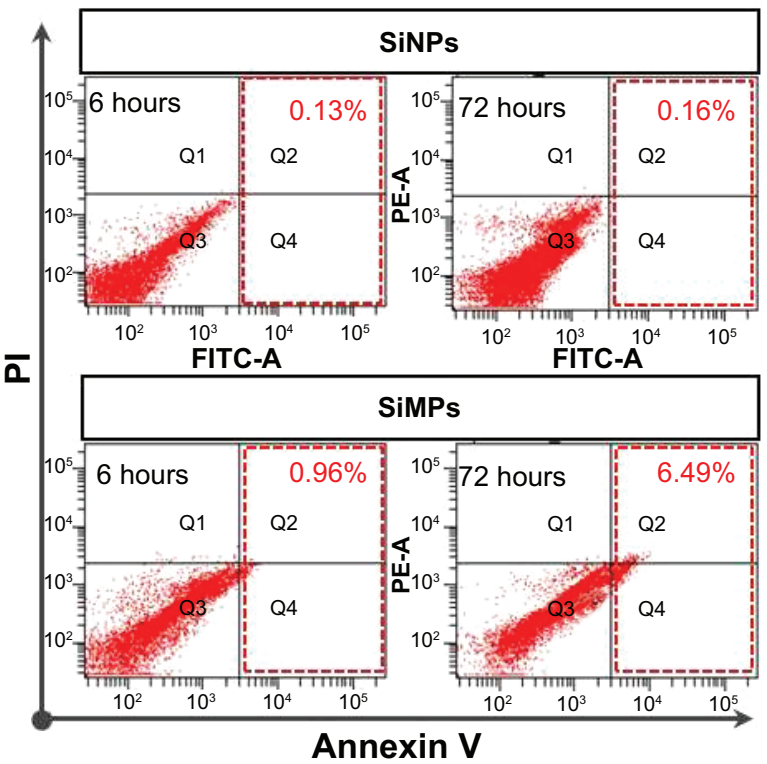

Figure 3 Proliferation and apoptosis assays in ADSCs treated with silica particles.

Notes: (A) Proliferation assay was performed by measuring DNA content. Total DNA (ng/ $\mu$ L) was increased in silica NPs with $1 \%$ FBS group compared with NP and MP media groups $(* P<0.00 I)$. Experiments were performed in at least triplicate. The data are representative of three independent experiments. (B) Induction of apoptosis in human ADSCs treated with silica NP medium or silica MP medium was detected by Annexin $V$ assay. ADSCs were treated with silica NPs or silica MPs for 6 and 72 hours after the cells were incubated in FBS-free medium for 24 hours. (C) DAPI staining of cells incubated in each type of medium for 24 hours. White arrows indicate DNA fragmentation in silica MPs.

Abbreviations: ADSCs, adipose tissue-derived stem cells; DAPI, 4,6-diamidino-2-phenyllindile; FBS, fetal bovine serum; NPs, nanoparticles; MPs, microparticles; SiNPs, silicon nanoparticles; SiMPs, silicon microparticles; PI, propidium iodide; FITC, fluorescein isothiocyanate.

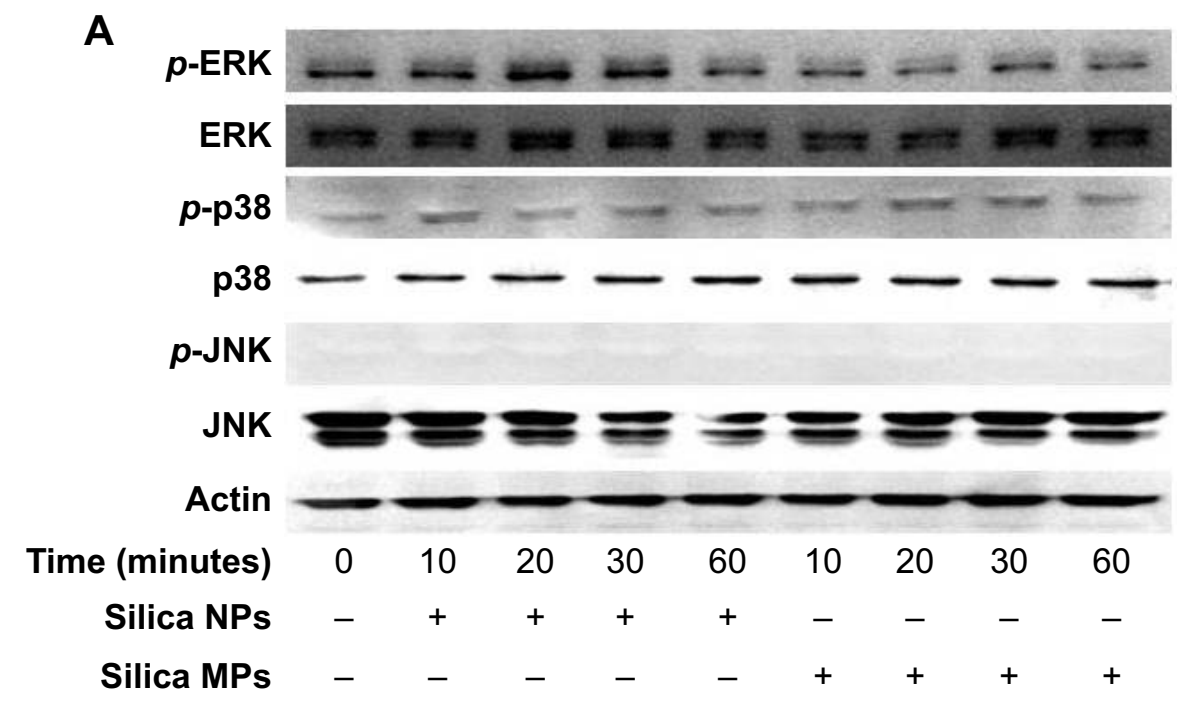

Figure 4 (Continued) 


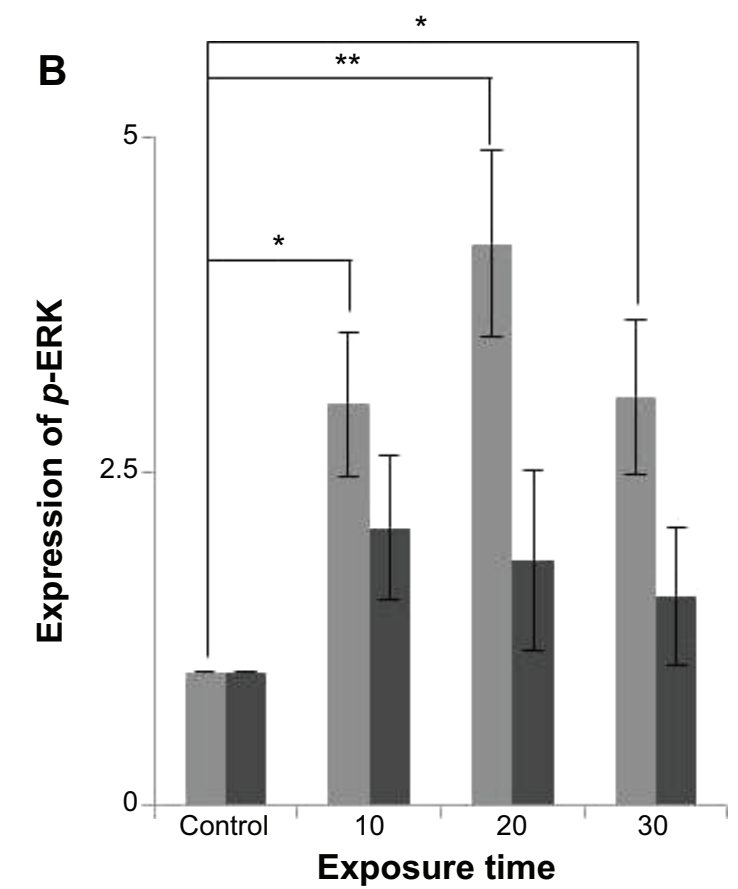

D

\section{p-ERK}

ERK

\section{Actin}

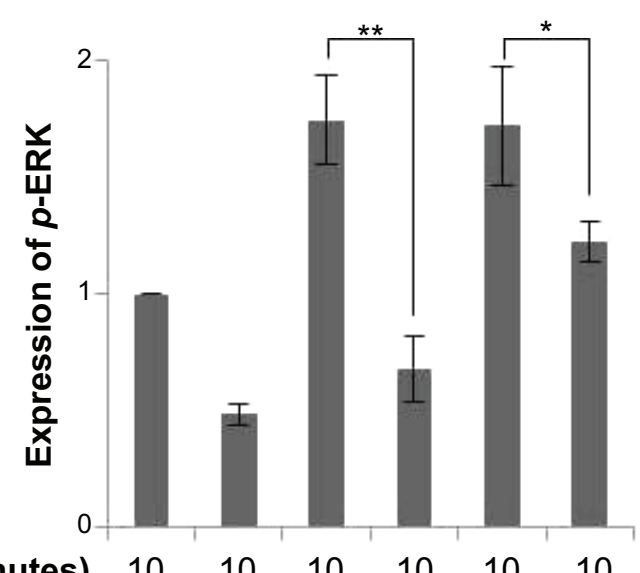

$\begin{array}{lllllll}\text { Time (minutes) } & 10 & 10 & 10 & 10 & 10 & 10\end{array}$

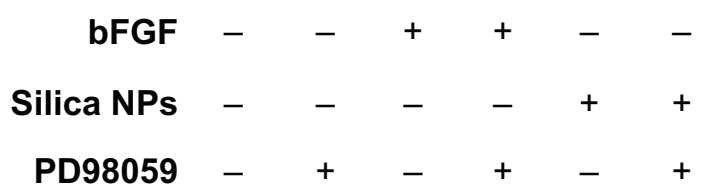

C

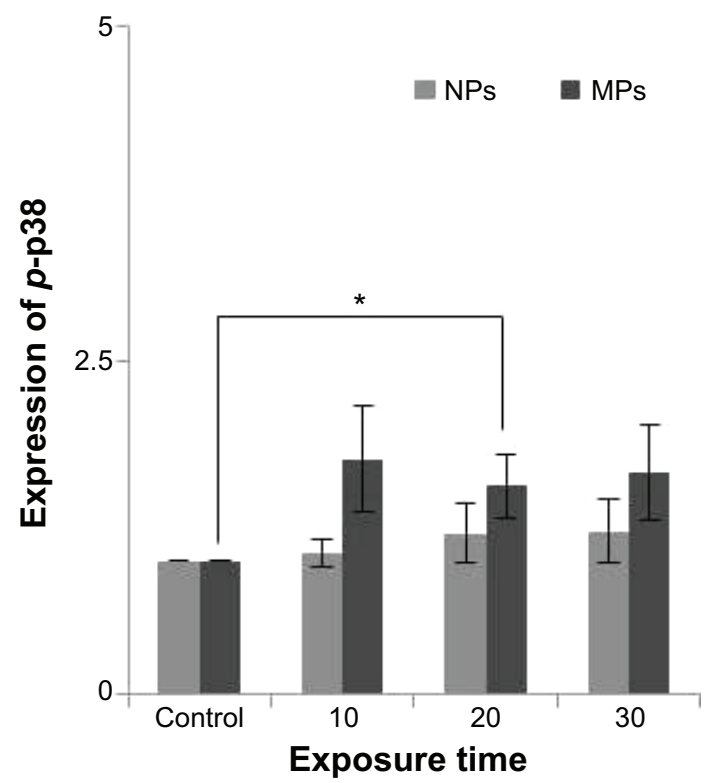

E

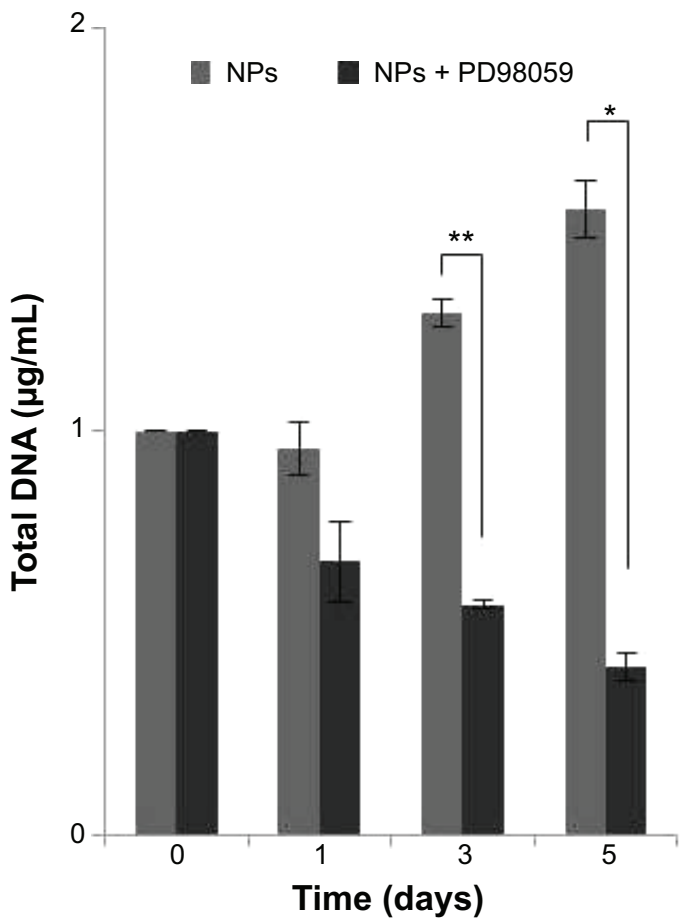

Figure 4 Activation of mitogen-activated protein kinases by differently sized silica particles.

Notes: (A) Protein extracts from the cells incubated in each type of medium for 10, 20, 30 and 60 minutes after starving in FBS-free medium for 24 hours were immunoblotted. (B, C) Quantitative analysis of ERKI/2 and $\mathrm{p} 38$ phosphorylation level by densitometric analysis. Three independent experiments were performed $(* P<0.01$, $* * P<0.001$ ). (D) ERKI/2 activation by silica NPs was evaluated by treatment with PD98059, a MEK-ERK inhibitor. Protein was extracted from cells pretreated with PD98059 for 30 minutes, followed by treatment with silica NPs for 10 minutes. Quantitative analysis of three independent experiments by densitometric analysis was performed. ERK I/2 phosphorylation by basic fibroblast growth factor and silica NPs was prevented by pretreatment with PD98059 ( $* P<0.0$ I, $* * P<0.00$ I). (E) A proliferation assay was performed by measuring DNA content in cells pretreated with PD98059 followed by treatment with silica NPs. Total DNA (ng/ $\mu \mathrm{L}$ ) was increased in the silica NPs with I\% FBS group, whereas the pretreated group showed reduced proliferation. Experiments were performed in triplicate at least.

Abbreviations: ERK, extracellular signal-related kinase; JNK, Janus kinase; NPs, nanoparticles; MPs, microparticles; bFGF, basic fibroblast growth factor; p-, phosphorylated; MEK, mitogen-activated protein kinase kinase; FBS, fetal bovine serum. 


\section{Discussion}

Many studies have shown that silica-based scaffolds and silica-coated plates induce cell proliferation, attachment, and biocompatibility. ${ }^{24-27}$ In previous reports, silicon has been shown to be released into culture medium and to induce cell proliferation. ${ }^{28,29}$ Interestingly, we found that silica NPs (around 50-120 nm in size) have effects on MAPK signaling and cell proliferation that were different from those of silica MPs (around 2-4 $\mathrm{m}$ in size). Consistent with our observations, ceramic NPs have been reported to stimulate cell growth to a greater extent than MPs. ${ }^{30}$ Protein adsorption is also enhanced on nanostructured silicon, ${ }^{31}$ and nano-features increase the formation and adhesive strength of fibroblast cells. ${ }^{32}$

Silica-based scaffolds ${ }^{33}$ and coated plates ${ }^{34}$ have hydrophilic properties. When we examined the interaction of silica-NP or silica-MP with ADSCs, only silica NPs entered the cells and some were clustered in the vesicles, suggesting that silica NPs enter the cells in part through endocytosis. Of note, no particles were observed in the nucleus (Figure 2E and F). Additionally, Figure S3 shows endocytosis of silicaNPs that have been entering into the cytosol of cell. This finding indicates that silica NPs in vesicles and the cytosol may affect signaling pathways. ${ }^{17,18}$ In contrast, silica MPs were not transferred to the cytosol (Figure 2D), suggesting that their particle size might have impeded access. Interestingly, we found that silica NPs increased growth of hADSCs, whereas silica MPs induced apoptosis (Figure 3). Therefore, it is possible that endocytosis of silica NPs accelerates cell growth whereas cell membrane stress caused by silica MPs induces apoptosis. ${ }^{35}$

To further address the mechanism of action of these differentially sized particles, we examined the effects of silica NPs and MPs on the MAPK pathway (Figure 4A). Involvement of ERK1/2 signaling in cell growth has been reported previously. ${ }^{36}$ Exposure of cells to silica NP medium initially resulted in potent activation of ERK1/2 signaling, followed by a return to basal levels (Figure 4B). Although the results of this experiment cannot be directly introducing exocytosis but cell metabolism was circulated according to endocytosis and exocytosis. ${ }^{37}$ Silica NPs had no effect on phosphorylation of JNK (Figure S4B), which is involved in apoptosis and inflammation. ${ }^{38,39}$ The $\mathrm{p} 38$ signaling pathway is related to cell stress and apoptosis. ${ }^{36,39}$ Consistent with previous reports, the $\mathrm{p} 38$ signaling pathway was not affected significantly by silica NPs, whereas silica MPs increased p38 phosphorylation levels (Figure 4C).

We confirmed the effect of silica NPs on ERK signaling by inhibiting MEK, an upregulator of ERK signaling. The proliferation-related MEK-ERK1/2 signaling pathway is suppressed by PD98059 via MEK signaling inhibition, ${ }^{40}$ while phosphorylation of JNK and p38 signaling are unaffected. ${ }^{38}$ Our results showed that PD98059 dosedependently decreased phosphorylation of ERK in the silica NP medium (Figure S4A). Thus, the data indicate that PD98059 inhibits MEK-ERK1/2 signaling driven by silica NPs. Consistent with the proliferation effect of silica NPs, PD98059 also suppressed the proliferation of

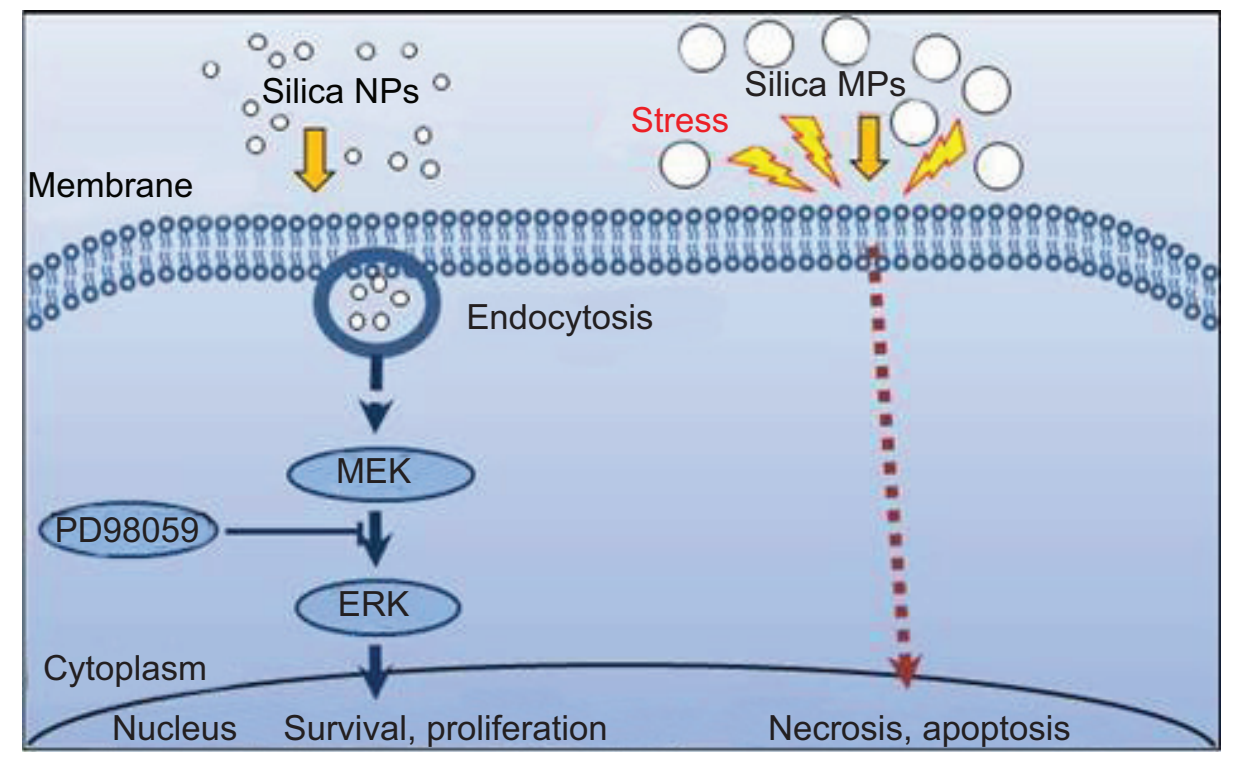

Figure 5 Proposed signaling pathways affected by silica NPs and silica MPs.

Abbreviations: NPs, nanoparticles; MPs, microparticles; ERK, extracellular signal-related kinase; MEK, mitogen-activated protein kinase kinase. 
hADSCs stimulated by silica NPs (Figure 4E). Together, the results indicate that silica NPs induce cell proliferation by activating the ERK1/2 signaling pathway (Figure 5). Attenuation and stabilization of growth factor signaling by endocytic control has been shown. ${ }^{18}$ It is still unclear whether endocytosis of silica NPs affects activation of ERK. In future, we will evaluate the signaling pathways involved in the multidifferentiation potency of hADSCs containing silica-NP and MP products.

\section{Conclusion}

Our results confirm that silica NPs enter the cytosol through the cell membrane by endocytosis, and silica NPs have accelerated cell growth. These data suggest that a silica NP size in the range of $50-120 \mathrm{~nm}$ is more effective in promoting stem cell growth by stimulating the ERK signaling pathway and that silica MPs induce apoptosis. But, this result was uncertain, when silica MP induced phosphorylation of P38. We will identify to the relevance of silica MPs and apoptosis as a future study. Therefore, selection of silica particle size is important when producing a scaffold used in tissue engineering and cell therapy.

\section{Acknowledgments}

This work was supported financially by the Seoul R\&BD Program of the Seoul Government of Korea(SS110011C0211601) and by a National Research Foundation of Korea grant funded by the Government of Korea.

\section{Disclosure}

The author reports no conflicts of interest in this work.

\section{References}

1. Moura J, Teixeira LN, Ravagnani C, et al. In vitro osteogenesis on a highly bioactive glass-ceramic (biosilicate). J Biomed Mater Res A. 2007;82:545-557.

2. Wu C, Chang J, Wang J, et al. Preparation and characteristics of a calcium magnesium silicate (bredigite) bioactive ceramic. Biomaterials. 2005;26:2925-2931.

3. Zhou H, Wei J, Wu X, et al. The bio-functional role of calcium in mesoporous silica xerogels on the responses of osteoblasts in vitro. J Mater Sci Mater Med. 2010;21:2175-2185.

4. Murphy S, Wren AW, Towler MR, et al. The effect of ionic dissolution products of $\mathrm{Ca}-\mathrm{Sr}-\mathrm{Na}-\mathrm{Zn}$-Si bioactive glass on in vitro cytocompatibility. J Mater Sci Mater Med. 2010;21:2827-2834.

5. Shi X, Wang Y, Varshney RR, et al. In-vitro osteogenesis of synovium stem cells induced by controlled release of bisphosphate additives from microspherical mesoporous silica composite. Biomaterials. 2009;30:3996-4005.

6. Farmer VC. Sources and speciation of aluminium and silicon in natural waters. Ciba Found Sympos. 1986;121:4-23.

7. Reffitt DM, Ogston N, Jugdaohsingh R, et al. Orthosilicic acid stimulates collagen type 1 synthesis and osteoblastic differentiation in human osteoblast-like cells in vitro. Bone. 2003;32:127-135.
8. Chen QZ, Thompson ID, Boccaccini AR. 45S5 Bioglass-derived glass-ceramic scaffolds for bone tissue engineering. Biomaterials. 2006;27:2414-2425.

9. Webster TJ, Patel AA, Rahaman MN, et al. Anti-infective and osteointegration properties of silicon nitride, poly(ether ether ketone), and titanium implants. Acta Biomater. 2012;8:4447-4454.

10. Wiens M, Wang X, Schlossmacher U, et al. Osteogenic potential of biosilica on human osteoblast-like (SaOS-2) cells. Calcif Tissue Int. 2010;87:513-524.

11. Shie MY, Chang HC, Ding SJ. Effects of altering the $\mathrm{Si} / \mathrm{Ca}$ molar ratio of a calcium silicate cement on in vitro cell attachment. Int Endod J. 2012;45:337-345.

12. Zhang $\mathrm{M}$, Wu $\mathrm{C}$, Lin $\mathrm{K}$, et al. Biological responses of human bone marrow mesenchymal stem cells to $\mathrm{Sr}-\mathrm{M}-\mathrm{Si}(\mathrm{M}=\mathrm{Zn}, \mathrm{Mg})$ silicate bioceramics. J Biomed Mater Res A. 2012;100:2979-2990.

13. Jin Z, Gong Y-Q, Qin X-C, et al. Biodegradable poly(lactic-coglycolic acid) Maxpol-T/S as novel scaffold for adipose derived stem cells and fibroblast growth in vitro. Tissue Eng Regen Med. 2008;5: 869-876.

14. Lee JH, Lee KH, Kim MH, Kim JP, Lee SJ, Yoon J. Possibility of undifferentiated human thigh adipose stem cells differentiating into functional hepatocytes. Arch Plast Surg. 2012;39:593-599.

15. Xia L, Kin K, Jiang X, et al. Effect of nano-structured bioceramic surface on osteogenic differentiation of adipose derived stem cells. Biomaterials. 2014;35:8514-8527.

16. Kang KS, Hong JM, Jeong YH, et al. Combined effect of three types of biophysical stimuli for bone regeneration. Tissue Eng Part A. 2014;20:1767-1777.

17. Oh NR, Park JH. Endocytosis and exocytosis of nanoparticles in mammalian cells. Int J Nanomedicine. 2014;9:51-63.

18. Dobrowolski R, De Robertis EM. Endocytic control of growth factor signalling: multivesicular bodies as signalling organelles. Nat Rev Mol Cell Biol. 2012;13:53-60.

19. Wang C, Lin K, Chang J, et al. Osteogenesis and angiogenesis induced by porous beta-CaSiO(3)/PDLGA composite scaffold via activation of AMPK/ERK1/2 and PI3K/Akt pathways. Biomaterials. 2013;34:64-77.

20. Schubert R, Geiger H, Zielen S, et al. Simultaneous detection of ERK-, p38, and JNK-MAPK phosphorylation in human adipose-derived stem cells using the cytometric bead array technology. J Immunol Methods. 2009;350:200-204.

21. Yang J, Zhang H, Zhao L, et al. Human adipose tissue-derived stem cells protect impaired cardiomyocytes from hypoxia/reoxygenation injury through hypoxia-induced paracrine mechanism. Cell Biochem Funct. 2012;30:505-514.

22. Kim BS, Jung JS, Jang JH, et al. Nuclear Argonaute 2 regulates adipose tissue-derived stem cell survival through direct control of miR10b and selenoprotein N1 expression. Aging Cell. 2011;10:277-291.

23. Lee JH, Rhie JW, Oh DY, et al. Osteogenic differentiation of human adipose tissue-derived stromal cells (hASCs) in a porous three-dimensional scaffold. Biochem Biophys Res Commun. 2008;370:456-460.

24. Brandao SM, Schellini SA, Moraes AD, et al. Biocompatibility analysis of bioglass(R) 45S5 and biosilicate(R) implants in the rabbit eviscerated socket. Orbit. 2012;31:143-149.

25. Xu C, Su P, Chen X, et al. Biocompatibility and osteogenesis of biomimetic bioglass-collagen-phosphatidylserine composite scaffolds for bone tissue engineering. Biomaterials. 2011;32:1051-1058.

26. Lu H, Kawazoe N, Tateishi T, et al. In vitro proliferation and osteogenic differentiation of human bone marrow-derived mesenchymal stem cells cultured with hardystonite (Ca2ZnSi 2O7) and \{beta\}-TCP ceramics. J Biomater Appl. 2010;25:39-56.

27. Thanyaphoo S, Kaewsrichan J. Synthesis and evaluation of novel glass ceramics as drug delivery systems in osteomyelitis. J Pharm Sci. 2012;101:2870-2882.

28. Zou S, Ireland D, Brooks RA, et al. The effects of silicate ions on human osteoblast adhesion, proliferation, and differentiation. J Biomed Mater Res B Appl Biomater. 2009;90:123-130. 
29. Sun J, Li J, Liu X, et al. Proliferation and gene expression of osteoblasts cultured in DMEM containing the ionic products of dicalcium silicate coating. Biomed Pharmacother. 2009;63:650-657.

30. Puckett S, Pareta R, Webster TJ. Nano rough micron patterned titanium for directing osteoblast morphology and adhesion. Int J Nanomedicine. 2008;3:229-241.

31. Sommerfeld J, Richter J, Niepelt R, et al. Protein adsorption on nanoscaled, rippled $\mathrm{TiO}_{2}$ and Si surfaces. Biointerphases. 2012;7:55.

32. Domanski M, Winnubst L, Luttge R, et al. Production and characterization of miro- and nano-features in biomedical alumina and zirconia ceramics using a tape casting route. J Mater Sci Mater Med. 2012;23: 1637-1644.

33. Tan F, Naciri M, Al-Rubeai M. Osteoconductivity and growth factor production by MG63 osteoblastic cells on bioglass-coated orthopedic implants. Biotechnol Bioeng. 2011;108:454-464.

34. Yun HS, Kim SH, Khang D, et al. Biomimetic component coating on 3D scaffolds using high bioactivity of mesoporous bioactive ceramics. Int J Nanomedicine. 2011;6:2521-2531.

35. Chan S, Chen MP, Cao JM, et al. Carvedilol protects against ironinduced microparticle generation and apoptosis of endothelial cells. Acta Haematol. 2014;132:200-210.
36. Payne KA, Meszaros LB, Phillippi JA, et al. Effect of phosphatidyl inositol 3-kinase, extracellular signal-regulated kinases $1 / 2$, and p38 mitogen-activated protein kinase inhibition on osteogenic differentiation of muscle-derived stem cells. Tissue Eng Part A. 2010;16:3647-3655.

37. Oh N, Park JH. Endocytosis and exocytosis of nanoparticles in mammalian cells. Int J Nanomedicine. 2014;9:51-63.

38. Ho PJ, Chou CK, Yeh SF. Role of JNK and p38 MAPK in Taiwanin A-induced cell death. Life Sci. 2012;91:1358-1365.

39. Widenmaier SB, Ao Z, Kim SJ, et al. Suppression of p38 MAPK and JNK via Akt-mediated inhibition of apoptosis signal-regulating kinase 1 constitutes a core component of the beta-cell pro-survival effects of glucose-dependent insulinotropic polypeptide. J Biol Chem. 2009;284:30372-30382.

40. Sarina, Yagi Y, Nakano O, et al. Induction of neurite outgrowth in PC12 cells by artemisinin through activation of ERK and p38 MAPK signaling pathways. Brain Res. 2013;1490:61-71. 


\section{Supplementry materials}
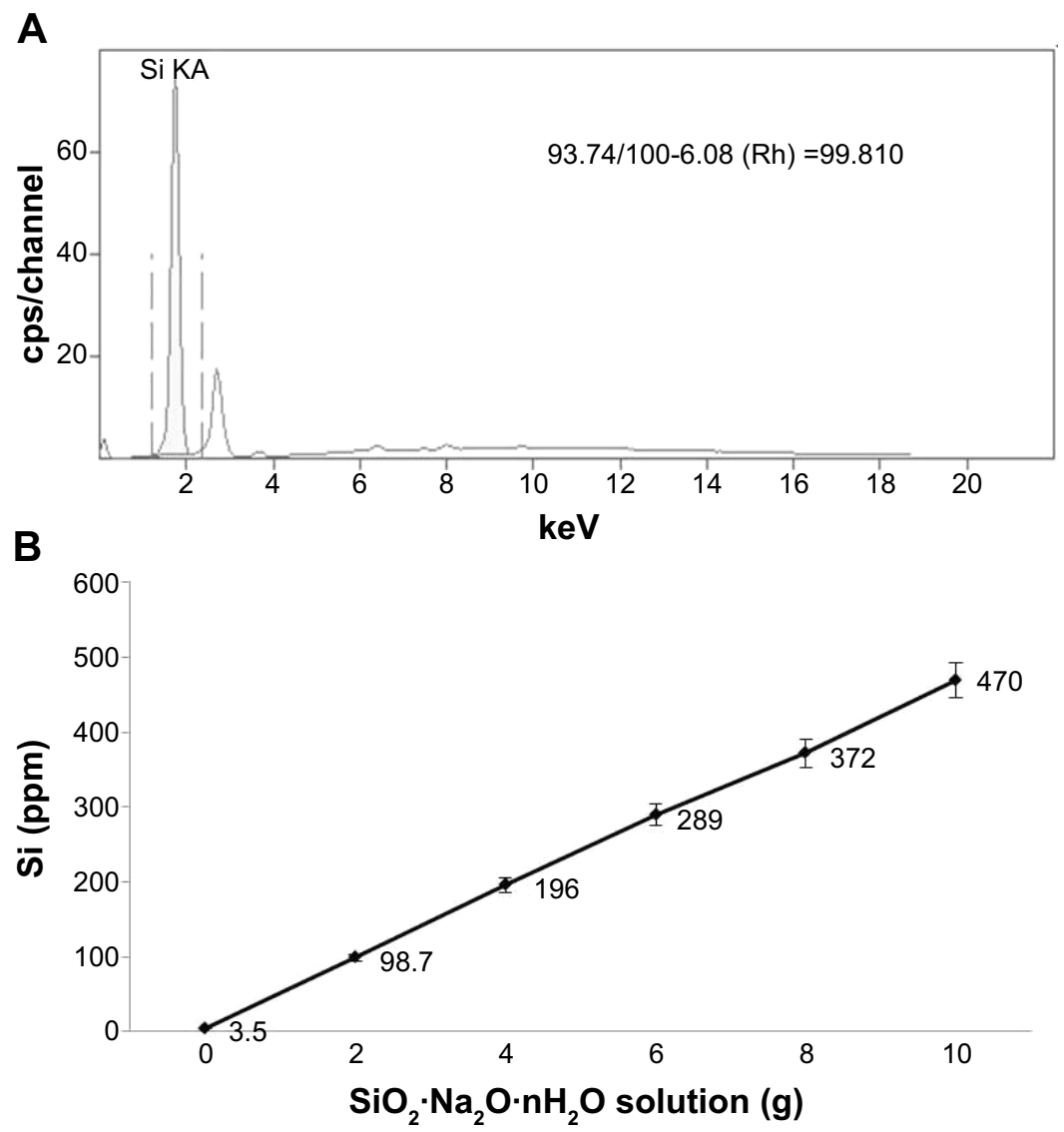

Figure SI Analysis of purified silicon dioxide (silica).

Notes: (A) Purity $(99.81 \%)$ of prepared silica gel was determined by X-ray fluorescence analysis. (B) Standard curve indicates that the amount of silicon is proportional to the amount of sodium silicate that was reacted with hydrochloric acid. The amount of silicon was analyzed by an inductively coupled plasma method.

A

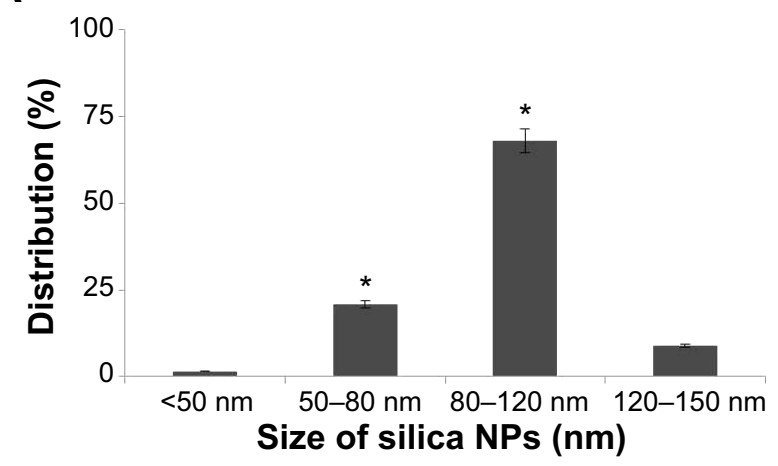

B

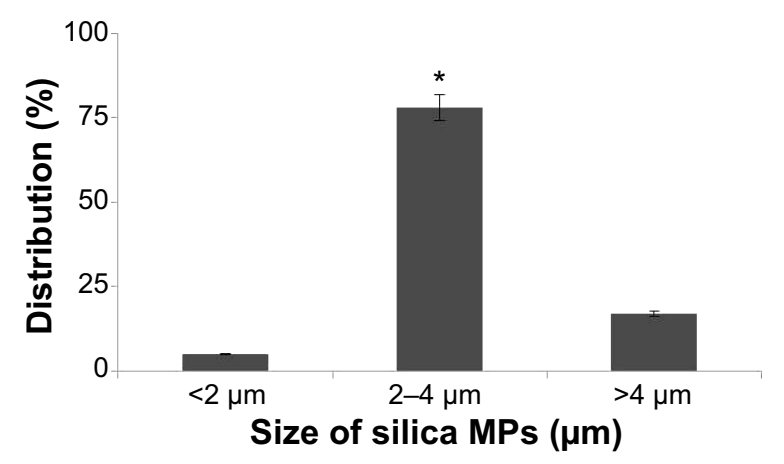

Figure S2 Particle distribution of silica NPs and silica MPs.

Notes: (A) Silica NP distribution according to size, ie, $<50 \mathrm{~nm}$ (I.6\%), 50-80 nm (2I\%), 80-120 nm (68\%), and I20-I50 nm (8.9\%). (B) Silica MP distribution according to size, ie, $<2 \mu \mathrm{m}(5 \%), 2-4 \mu \mathrm{m}(78 \%)$, and $>4 \mu \mathrm{m}(17 \%)(* P<0.0$ I).

Abbreviations: NPs, nanoparticles; MPs, microparticles. 

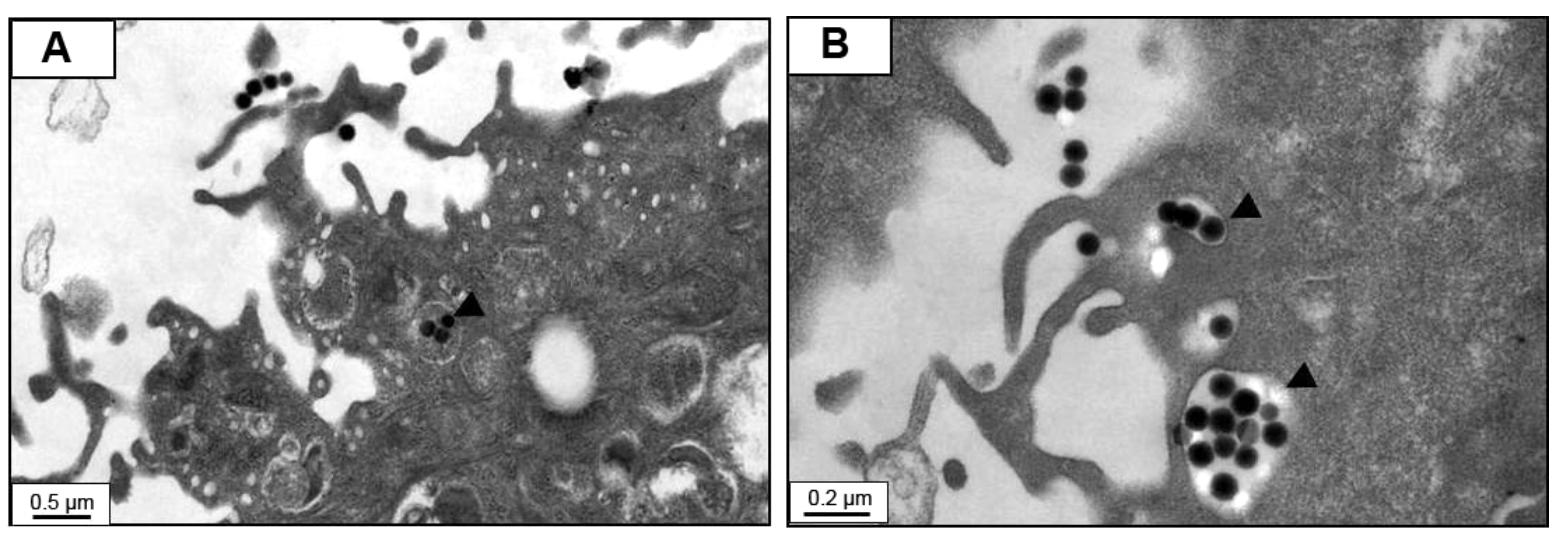

Figure S3 Transmission electron microscopic images of cells treated with silica nanoparticles.

Notes: $(\mathbf{A})$ and $(\mathbf{B})$ show cell membrane that vesicles entered to cytosol by endocytosis and triangle arrow indicates endocytosis of nanoparticles.

A

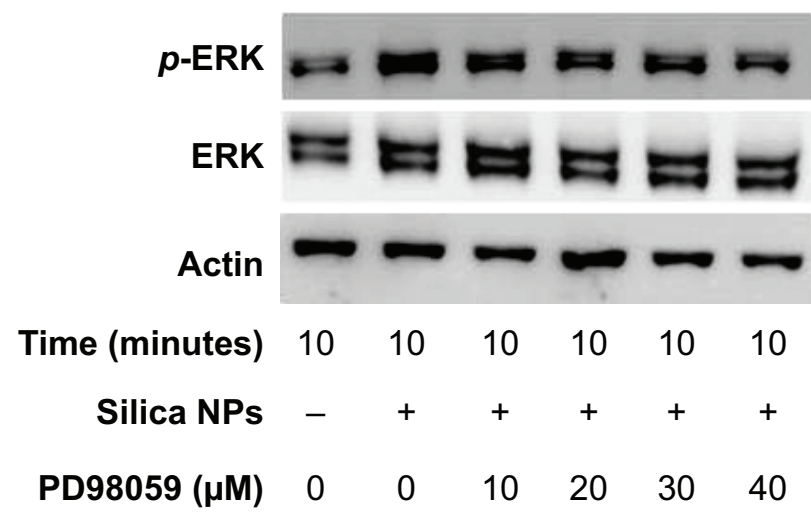

B

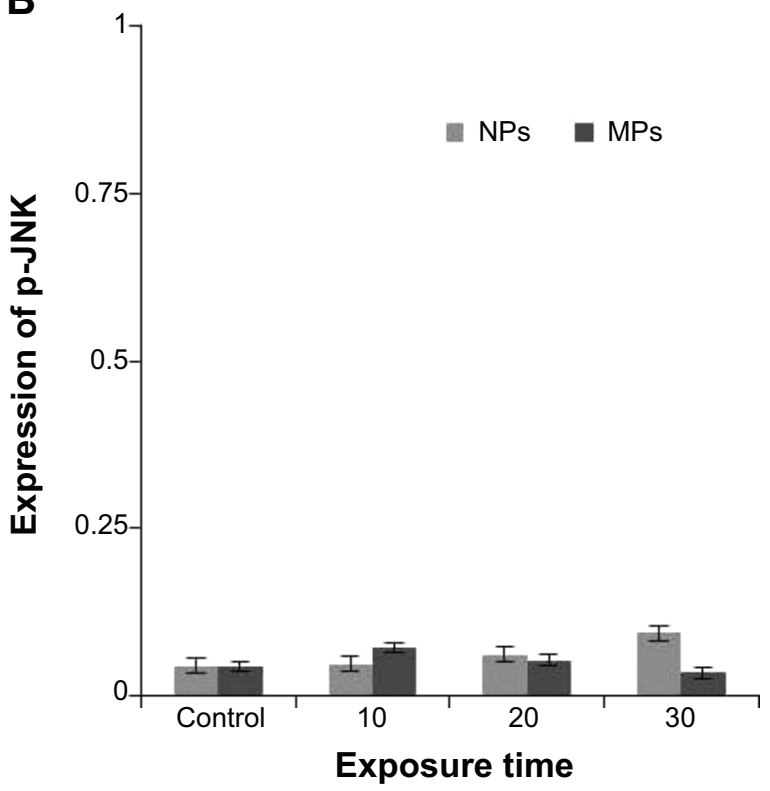

Figure S4 ERK and JNK Phosphorylation of hADSCs with silica NPs and MPs.

Notes: (A) Phosphorylation of ERK induced by silica NPs is inhibited by PD98059 in a dose-dependent manner. (B) Quantitative analysis of JNK phosphorylation level of ADSCs by densitometric analysis according to silica NPs and silica MPs. Reproduced with permission from Kim KJ, Jeon YJ, Lee JH, et al. The Effect of Silicon lon on Proliferation and Osteogenic Differentiation of Human ADSCs. Korea Tissue Engineering and Regenerative medicine. 20I0;7(2): 17I-I 177.'

Abbreviations: NPs, nanoparticles; MPs, microparticles; ERK, extracellular signal-related kinase; JNK, Jun amino-terminal kinases; PD98059, MEK-ERK inhibitor.

\section{Reference}

1. Kim KJ, Jeon YJ, Lee JH, et al. The Effect of Silicon Ion on Proliferation and Osteogenic Differentiation of Human ADSCs. Korea Tissue Engineering and Regenerative medicine. 2010;7(2):171-177.

\section{Publish your work in this journal}

The International Journal of Nanomedicine is an international, peerreviewed journal focusing on the application of nanotechnology in diagnostics, therapeutics, and drug delivery systems throughout the biomedical field. This journal is indexed on PubMed Central, MedLine, CAS, SciSearch ${ }^{\circledR}$, Current Contents ${ }^{\circledR} /$ Clinical Medicine,
Journal Citation Reports/Science Edition, EMBase, Scopus and the Elsevier Bibliographic databases. The manuscript management system is completely online and includes a very quick and fair peer-review system, which is all easy to use. Visit http://www.dovepress.com/ testimonials.php to read real quotes from published authors. 